Mots. Les langages du politique

$94 \mid 2010$

Trente ans d'étude des langages du politique

(1980-2010)

\title{
Parler en politique
}

\section{Christian Le Bart}

\section{(2) OpenEdition \\ Journals}

Édition électronique

URL : https://journals.openedition.org/mots/19867

DOI : $10.4000 /$ mots. 19867

ISSN : 1960-6001

Éditeur

ENS Éditions

\section{Édition imprimée}

Date de publication : 30 novembre 2010

Pagination : 77-84

ISBN : 978-2-84788-235-3

ISSN : 0243-6450

Référence électronique

Christian Le Bart, «Parler en politique », Mots. Les langages du politique [En ligne], 94 | 2010, mis en

ligne le 06 novembre 2012, consulté le 28 avril 2022. URL : http://journals.openedition.org/mots/

19867 ; DOI : https://doi.org/10.4000/mots. 19867 


\section{Mots \\ Les langages du politique}

№ 94 novembre 2010

\section{Trente ans d'étude des langages du politique (1980-2010)}

ouvrage coordonné par Paul BACOT, Marlène Coulomb-GulLY, Jean-Paul Honoré, Christian Le BART, Claire Oger, Christian Plantin

\section{SOMMAIRE}

Paul Bacot, Marlène Coulomb-Gully, Jean-Paul Honoré, Christian Le Bart, Claire Oger, Christian Plantin

Le discours politique n'est pas transparent. Permanence

et transformations d'un objet de recherche

OUTILS ET ENJEUX DU DISCOURS POLITIQUE

Ruth Amossy, Roselyne Koren

Argumentation et discours politique

Christian Plantin

Argumentation-rhétorique. Les eaux mêlées

Caroline Ollivier-Yaniv

Discours politiques, propagande, communication, manipulation 
Marc Bonhomme

La caricature politique

Paul Bacot

Développement et diversification d'une onomastique politique

Ruth Wodak

The Discursive Construction of History. Brief Considerations

Henri Boyer

Les politiques linguistiques

\section{LIEUX DE LA PRODUCTION DU DISCOURS POLITIQUE}

Christian Le Bart

Parler en politique

Dominique Maingueneau

Le discours politique et son «environnement»

Alice Krieg-Planque, Claire Oger

Discours institutionnels. Perspectives pour les sciences

de la communication

Sophie Béroud, Josette Lefèvre

Le corpus syndical. Une expérience au long cours

Corinne Gobin, Jean-Claude Deroubaix

L'analyse du discours des organisations internationales.

Un vaste champ encore peu exploré

François de la Bretèque

Le retour de la parole politique dans le cinéma français

Jean-Claude Soulages

Vie et mort du citoyen cathodique

Jacques Guilhaumou

Les discours de la Révolution française.

Aperçu d'ensemble d'un trajet de recherche (1980-2009) 


\section{DISCIPLINES ET CHAMPS DE RECHERCHE POUR L'ÉTUDE DES LANGAGES DU POLITIQUE}

Philippe Braud

L'apport de la science politique à l'étude des langages du politique

Claire Blandin

L'apport de l'histoire des médias à l'étude des langages du politique

Jean-François Tétu, Bernard Lamizet

Les SIC et les langages du politique

Sylvianne Rémi-Giraud

Sémantique lexicale et langages du politique.

Le paradoxe d'un mariage difficile?

Marlène Coulomb-Gully, Juliette Rennes

Genre, politique et analyse du discours.

Une tradition épistémologique française gender blind

Johannes Angermüller

Analyser le discours politique en Allemagne (1980-2010)

Érik Neveu

L'apport de Pierre Bourdieu à l'analyse du discours.

D'un cadre théorique à des recherches empiriques

Roselyne Ringoot

Questionner le discours avec Michel Foucault.

Actualisations théoriques et actualité éditoriale

\section{ENTRETIEN}

Maurice Tournier

Mots et politique, avant et autour de 1980

La revue Mots. Les langages du politique encourage l'usage des rectifications de l'orthographe proposées par le Conseil supérieur de la langue française et approuvées par l’Académie (Journal officiel, $n^{\circ}$ 100, 6 décembre 1990). 


\section{Christian Le Bart}

\section{Parler en politique}

Problématique centrale pour toute analyse de discours, la question de savoir qui parle est évidemment plus complexe qu'il n'y paraît. Formellement, bien sûr, le discours est imputable à un locuteur. Mais toute l'histoire des sciences sociales en général, des sciences du langage en particulier, plaide pour ne jamais en rester à cette pseudo-évidence. Freud, Marx, Durkheim, plus récemment Foucault ou Bourdieu (2001) n'ont cessé de nous mettre en garde contre une définition naïvement souverainiste de l'acteur social (et donc du locuteur). Le social est là qui le contraint (sûrement), le détermine (peut-être), dont la description ou même le simple repérage ouvre de nombreuses perspectives de recherche.

\section{Les déterminants de la parole politique}

S'agissant du discours politique, ces considérations trop générales prennent une tonalité particulière. La théorie de la démocratie représentative explicite en effet les contraintes qui encadrent le droit de parole conféré aux gouvernants : ceux-ci parlent au nom de représentés, ils parlent même à leur place, mais en retour ces derniers doivent se reconnaître dans ce qui est dit en leur nom. En ce sens, le discours politique est nécessairement, en démocratie, contraint. En même temps, et parce que les mandats politiques ne sont jamais impératifs, la théorie de la démocratie représentative tolère ce que la sociologie n'a fait, depuis, que constater : l'autonomie partielle des locuteurs. Le peuple ne parle pas, les institutions non plus, et ceux qui sont socialement habilités à s'exprimer en leur nom ne sont jamais complètement contraints. La représentation habilite, légitime et autorise autant qu'elle oblige et interdit. D'où la pertinence d'une approche plus stratégique que déterministe. Cette perspective a nourri toute une série de travaux sur la communication politique : comment les gouvernants parviennent-ils à travailler leur présentation de soi pour apparaître plus compétents, plus sympathiques, plus proches, plus volontaristes, etc.?

IEP de Rennes, CRAPE

lbrt35@club-internet.fr 
La professionnalisation de ce champ d'activité a consolidé la spécialisation concomitante des chercheurs ${ }^{1}$.

L'analyse de discours, telle que la propose Dominique Maingueneau (1991), est devenue au fil des recherches la référence fondamentale pour l'étude du discours politique. Elle permet la synthèse entre les perspectives déterministe et stratégique. Ainsi étudiera-t-on l'énonciation à la fois comme stratégie et comme production contrainte. Le repérage des contraintes qui encadrent la prise de parole politique est tendanciellement infini. Mais force est de constater que les analyses conduites ces dernières décennies, par les chercheurs français au moins, ont privilégié quelques angles d'attaque. Le premier est le format médiatique. La télévision a-t-elle changé les façons de faire de la politique? (Bonnafous éd., 1989; Tournier, Coulomb-Gully éd., 2001; Groupe de Saint-Cloud, 1995; Coulomb-Gully, 2001). Le Net est-il en train de transformer la politique? (Barbet, Bonhomme, Rinn éd., 2006) La seconde variable bien travaillée est le positionnement politique : discours politique de droite vs discours politique de gauche? On notera la richesse des travaux sur l'extrême droite (Souchard et al., 1997; Bonnafous, Fiala éd., 1999), sur le populisme (Périès, Taguieff éd., 1998). Le surgissement de l'extrême droite interpellait évidemment les chercheurs : mais le secret du vote Le Pen est-il dans le discours d'un leader souvent qualifié de tribun? Et peut-on, avec les armes des sciences sociales, dévoiler les artifices (pour ne pas dire les ficelles) d'une rhétorique que la communauté des chercheurs condamne? Du coup, les partis politiques plus classiques apparaissent délaissés, de même la perspective comparative qui, seule, pourrait permettre de vraiment tester l'hypothèse d'une singularité du discours d'extrême droite. La dernière entrée privilégiée est celle qui se centre sur les rôles. C'est sans surprise, dans le cas français, le rôle présidentiel qui retient l'attention. La longue tradition de recherche qui avait conduit les chercheurs à s’intéresser aux discours du général de Gaulle ou de François Mitterrand (Labbé, 1990) est reprise par Damon Mayaffre travaillant sur Jacques Chirac (Mayaffre, 2004).

Le bilan de ces travaux est extrêmement riche, mais pointe malgré tout une interrogation centrale quant à la hiérarchie des variables pertinentes et à la façon dont ces variables s'articulent : style personnel (Sarkozy vs Villepin)? Groupes d'appartenance du locuteur (les énarques, les représentants des minorités visibles, les femmes...) ? Rôle endossé (ministre, candidat à une élection présidentielle, maire...) ? Situation de communication (meeting, JT, travail parlementaire...) ? Contexte (campagne électorale, situation de crise...) ? Famille politique? Génération politique? On voit au passage les limites, bien théorisées par Giddens, d’une opposition naïve entre contraintes et intentionnalité

1. Il faudrait ici citer les travaux d’Érik Neveu, de Jean-Baptiste Legavre, de Pierre Leroux, de Philippe Teillet, de Philippe Riutort... Parmi quelques synthèses : Curapp, 1991; Le Bart, 1998; Charaudeau, 2005 ; Bonnafous et al., 2003; Gerstlé, 2004 ; Riutort, 2007. 
stratégique. Le métier politique consiste précisément à savoir poursuivre des stratégies discursives (convaincre, se légitimer, délégitimer autrui...) en ayant intégré l'ensemble des contraintes de champ. C'est l'intériorisation du rôle.

On prolongera cette synthèse partielle (et sans doute partiale) en suggérant deux pistes de recherche qui permettent d'articuler détermination par les logiques de champ et stratégies d'acteur.

\section{Parler pour exister}

L'illusion du locuteur auteur de son discours n'est pas seulement pour le chercheur un obstacle à surmonter; elle est aussi un objet à étudier, en particulier du fait des conséquences (objectives) qu'elle produit dans le champ politique. Plus que jamais le discours politique fait exister un champ politique individualisé, c'est-à-dire peuplé de locuteurs stratèges en compétition les uns avec les autres. Le discours politique contribue directement à l'individualisation du champ politique par les jeux d'imputation au locuteur, et à lui seul, de ce qui est dit. Quand, a contrario, la décision apparaît de plus en plus en plus coproduite (et même si les « décideurs » politiques tentent de donner leur nom à telle ou telle réforme), le discours est rigoureusement attribué au locuteur par les commentateurs autorisés (autres politiques, journalistes, observateurs de la vie politique). Les politiques existent plus que jamais à travers ce qu'ils disent, la capacité à exister à travers ce qu'ils font apparaissant beaucoup plus aléatoire (ce qui n'est pas pour rien dans ce qu'il est convenu d'appeler la crise de la représentation). Difficile pour un professionnel de ne pas assumer la responsabilité de propos tenus dans l'exercice de son rôle, comme le montre la gestion toujours très délicate des gaffes et écarts de langage.

L'imputation du discours au locuteur joue plus souvent dans un sens positif : c'est l'image classique du politique qui parle bien, qui convainc, qui parvient à séduire. Le charisme est attribué comme une qualité personnelle, il est censé exprimer une "nature», malgré ce que l'on peut savoir par ailleurs de son mode de construction (socialisation familiale, passage par les grandes écoles, média-training, influence des conseillers en communication, coproduction des argumentaires, des petites phrases ou même des discours, etc.). Face au public d'un meeting, face aux journalistes, face à ses contradicteurs, le politique apparaît seul; c'est lui et lui seul qui parle, c'est d'abord lui qui tirera profit des verdicts positifs qui sanctionneront son discours, c'est d'abord lui qui pâtira des éventuels dérapages. Le politique fait moins le discours que le discours ne fait le politique : Robert Badinter ou Simone Veil existent dans la mémoire collective par les plaidoyers par lesquels ils ont su faire avancer de grandes causes (abolition de la peine de mort, légalisation de l'avortement) : les lois qui portent leur nom sont supposées avoir été rendues possibles par 
leur capacité à convaincre, au-delà donc des simples logiques de discipline de vote. Dominique de Villepin fut longtemps l'homme du discours de l'ONU, Catherine Trautmann ou Michel Noir sont nés à la vie politique nationale par des prises de position fermes contre le Front national, FN dont le leader a luimême joué de la formule choc comme seul moyen d'exister pour une petite formation partisane. La mémoire politique associe volontiers telle figure politique et telle formule, au prix de réductions contre lesquelles les intéressés se rebellent volontiers: Michel Rocard n'en finit pas de s'expliquer sur «la France qui ne peut accueillir toute la misère du monde », Alain Juppé regrette de s'être dit «droit dans ses bottes » 2 .

Tout concourt donc à faire du discours le support fondamental des stratégies politiques. Les journalistes politiques accréditent cette vision en donnant à voir la vie politique principalement à travers des citations, laissant dans l'ombre les autres dimensions3. L'exemple américain invite aux mêmes conclusions : les travaux sur la présidentialisation rhétorique montre comment un acteur faiblement pourvu en ressources institutionnelles a pu s'affirmer au fil de l'histoire grâce à la place occupée dans les médias. Le président américain est d'abord un homme qui parle aux Américains (Dorsey, 2002 ; Friedenberg, 2002 ; Parry-Giles, 2002).

Les remarques précédentes peuvent nourrir une analyse en termes de stratégie individuelle, à condition de replacer ces stratégies elles-mêmes dans un contexte élargi d'individualisation du champ politique. Car c'est à de véritables injonctions que les politiques sont soumis : ils doivent, pour exister politiquement, publier, se faire inviter sur les plateaux de télévision, faire entendre une différence qui est au final constitutive de leur singularité... On peut pareillement articuler stratégie et déterminisme sur le terrain de la légitimation.

\section{Les grandeurs collectives. Parler pour se légitimer}

On se contentera ici de tenter de recenser les points d'appui à partir desquels les professionnels de la politique tentent de stabiliser leur prétention à monopoliser la parole politique légitime, celle auprès de laquelle le discours pro-

2. Point limite de ces logiques d'imputation du discours au seul locuteur : il est cependant intéressant de voir que le rôle de speech writer assumé par Henri Guaino auprès de Nicolas Sarkozy a acquis ces dernières années une forte visibilité médiatique et politique. Le mythe du président locuteur souverain en est-il affaibli? Ce n'est pas sûr, alors même qu'on a pu voir certaines polémiques nées de discours présidentiels mettre en cause l'auteur réel de tel ou tel énoncé (Henri Guaino, donc) et non le locuteur formel (Nicolas Sarkozy). Voir par exemple la critique, par Bernard-Henry Lévy, du discours de Dakar du 26 juillet 2007.

3. En contexte «civilisé » au sens d'Elias, c'est-à-dire lorsque la conquête du pouvoir ne se fait plus par l'exercice de la violence, la vie politique n'est-elle que discours? Une alternative au moins existe à cette réduction : la politique se fait aussi avec le corps, qui exprime, qui se déplace, dont la grammaire n'est donc pas réductible à la parole (Le Bart, 1998). 
fane, celui des citoyens par exemple, est toujours entaché de fragilité originelle. La grandeur des politiques est multiple, elle emprunte à plusieurs registres, elle se recompose au gré des crises du politique et des transformations sociétales. On peut dire que les politiques font en la matière feu de tout bois, qu'ils savent puiser dans tous les registres de légitimité disponibles. Ou, pour glisser du langage stratégique au langage déterministe : qu'ils se doivent de composer avec les grandeurs en circulation dans l'espace social, qu'ils doivent s'adapter aux changements idéologiques en adossant leur prétention à gouverner aux idéologies légitimatrices dominantes.

La première grandeur politique est élective. Les élus parlent au nom (et pas seulement à la place) de leurs électeurs. Ils bénéficient là d'un monopole de principe, celui du droit à parler du point de vue de la nation (pour les parlementaires par exemple). Pour un président de la République, ou à une autre échelle, un maire ou un président de région, la logique est autant celle de l'incarnation que de la représentation. Incarnation d'un territoire et d'une communauté, l'un et l'autre devenant par la magie sociale du suffrage universel des entités réelles, parlantes et agissantes (décidantes). Cette légitimité première, sans doute affaiblie aujourd'hui, reste fondamentale pour comprendre l'économie symbolique du champ politique. Elle est la légitimité originelle des gouvernants, celle qui leur permet de prendre la parole et de parler en surplomb. C'est cette légitimité qui autorise les politiques à dire «nous» en parlant au nom des individus. Ce faisant, ces mêmes politiques font exister comme autant de communautés des entités à la consistance sociologique très variable : une nation, une ville, une région... La performativité nominale (Paul Siblot) qui fonde des énoncés du type : «La France souhaite... », «La France n'acceptera pas... » repose en dernière instance sur une culture de « remise de soi » (Bourdieu) au terme de laquelle les représentants sont autorisés à parler à la place des représentés avec le consentement de ces derniers. Façon aussi de renvoyer aux inégalités scolaires, culturelles, et à tous les mécanismes qui creusent les différences entre élus et simples citoyens.

Les politiques ont longtemps ajouté à ce premier registre un second registre de légitimité, plus partisan. Comme précédemment, se réclamer du socialisme, du gaullisme, du communisme, oblige et autorise. Chaque formation politique ou chaque famille de pensée se caractérise par un lexique partiellement spécifique, une grammaire argumentative, une façon de mettre en mots et en récits le monde social en faisant exister des entités (la France, la classe ouvrière, le peuple...), en mobilisant une mémoire spécifique, en désignant des cibles, en sacralisant des valeurs. Il est banal de constater l'affaiblissement du poids de cette variable aujourd'hui, en particulier du côté des formations politiques les plus directement en mesure d'exercer le pouvoir. La différenciation discursive s'observe aux extrêmes (droite et gauche), tout se passant comme si les formations plus classiques empruntaient au même langage politique, voire 
développaient le même discours politique. On peut, dans le cas français, expliquer cette standardisation (relative) par l'accélération des alternances politiques nationales (et locales) : la culture de gouvernement, largement imprégnée des standards européens et internationaux, s'impose aux décideurs où qu'ils soient et quels qu'ils soient. Faut-il croire que les idéologies partisanes n'ont plus aucune légitimité? Les professionnels de la politique semblent avoir complètement intériorisé l'idée selon laquelle les marqueurs idéologiques étaient peu à même de susciter l'adhésion du corps électoral.

Le registre de l'efficacité et du pragmatisme s'est imposé comme forme dépolitisée du discours politique. Le paradoxe n'est qu'apparent : parce qu'il émane des gouvernants et qu'il vise à conforter leur légitimité, ce discours est aussi politique que les précédents. Mais il semble cesser de l'être dès lors qu'il n'emprunte plus aux formes traditionnellement constitutives de la rhétorique partisane. Les marqueurs idéologiques s'effacent ou s'euphémisent, le lexique est plus technocratique. Deux sources l'alimentent. La première est constituée par les professionnels de l'action publique : élites administratives, hauts fonctionnaires, experts, consultants, universitaires, produisent, depuis des lieux multiples (publics ou privés, étatiques, intra-étatiques, supra-étatiques), une idéologie de la gouvernance pragmatique qui réfère l'action publique à un idéal managérial standardisé et mondialisé. Cette imposition par en haut (top-down) ne suffirait pas à convertir les politiques. Mais ces derniers se trouvent également soumis à une pression ascendante (bottom-up) émanant de citoyens moins portés sur les lectures idéologiques et davantage disposés à apprécier une offre politique précise, concrète, pragmatique. Triomphe de l'idéologie néolibérale? Triomphe de l'idéologie de la proximité et du quotidien? Le discours politique n'est pas moins idéologique que par le passé, mais il est comme habité par les idéologies dominantes du moment (Boltanski, Chiapello, 1999). Les politiques mobilisent celles-ci en parlant « demande sociale», « démocratie participative », «bonne gouvernance», «évaluation »...

Il est incontestable que les idéologies partisanes sont aujourd'hui, pour une partie significative de l'électorat, associées à des périodes révolues de notre histoire politique. À l'inverse, la culture du diagnostic, de l'expertise de terrain et de l'évaluation s'impose sur le mode de l'évidence. La « réalité des choses » est le référent ultime auquel s'adosse un discours politique plus que jamais obsédé par le constat, l'observation. Cette sociologisation du discours politique demeure évidemment très superficielle. S’il faut prendre au sérieux la professionnalisation des politiques et noter l'augmentation objective de leur culture juridique, économique, managériale, voire sociologique, il faut aussi rappeler que le réalisme politique, comme le réalisme littéraire, est avant tout un style, un ton, une série de conventions par lesquelles un discours travaille à sa propre légitimation (et à celle de son auteur). Le fétichisme du constat, chiffres en main, la comparaison internationale pour évaluer sans a priori les 
solutions possibles et pour repérer les plus efficaces, la mise en scène des vrais gens rencontrés sur le terrain et ayant livré leur sentiment sincère ou ayant décrit leur situation réelle, tout cela relève moins de la conversion des politiques à la démarche des sciences sociales que de ce que Barthes appelait l'effet de réel. Les politiques devaient hier référer leurs prises de positions à un canon idéologique; ils doivent désormais prendre appui sur une supposée réalité sociale.

\section{Références}

Barbet D., Bonhomme M., Rinn M. éd., 2006, Mots. Les langages du politique, no80, mars, La politique mise au Net.

Bonnafous S. éd., 1989, Mots. Les langages du politique, n²0, septembre, La politique à la télévision.

Bonnafous S., Fiala P. éd., 1999, Mots. Les langages du politique, nº58, mars, Argumentations d'extrême droite.

Bonnafous S., Chiron P., Ducard D., Lévy C. éd., 2003, Argumentation et discours politique, Rennes, Presses universitaires de Rennes.

BOURDIEU P., 2001, Langage et pouvoir symbolique, Paris, Le Seuil.

Boltanski L., Chiapello E., 1999, Le nouvel esprit du capitalisme, Paris, Gallimard.

Charaudeau P., 2005, Le discours politique, Paris, Vuibert.

Coulomb-Gully M., 2001, La démocratie mise en scènes. Télévision et élections, Paris, CNRS.

CURAPP, 1991, La communication politique, Paris, PUF.

DORSEY L. éd., 2002, The Presidency and Rhetorical Leadership, Texas University Press, 2002.

Friedenberg R., 2002, Notable Speeches in Contemporary Presidential Campaigns, New York, Praeger.

GERSTLÉ J., 2004, La communication politique, Paris, Armand Colin.

Groupe de Saint-Cloud, Présidentielle. Regards sur les discours télévisés, Paris, INANathan, 1995.

LABBÉ D., 1990, Le vocabulaire de François Mitterrand, Paris, Presses de la FNSP.

LE BART C., 1998, Le discours politique, Paris, PUF (Que sais-je ?).

- 2009, "L'analyse des livres politiques», Questions de communication, nº15, p. 323-344.

Maingueneau D., 1991, L'analyse du discours, Paris, Hachette.

MAYAFFRE D., 2004, Paroles de président. Jacques Chirac et le discours présidentiel sous la Ve République, Paris, Champion.

PARrY-Giles S., 2002, The Rhetorical Presidency, Propaganda and the Cold War, 19441955, New York, Praeger.

PÉriès G., TAguieff P.-A. éd., 1998, Mots. Les langages du politique, nº55, juin, Discours populistes. 


\section{CHRISTIAN LE BART}

RIUTORT Ph., 2007, Sociologie de la communication politique, Paris, La Découverte.

Souchard M., Wahnich S., Cuminal I., Wathier V., 1997, Le Pen. Les mots, Paris, Le Monde Éditions.

Tournier M., Coulomb-Gully M. éd., 2001, Mots. Les langages du politique, nº67, décembre, La politique à l'écran : l'échec? 